\title{
A sensing mechanism for the detection of carbon nanotubes using selective photoluminescent probes based on ionic complexes with organic dyes
}

\author{
Petro Lutsyk ${ }^{1,2}$, Raz Arif ${ }^{2,3}$, Jan Hruby ${ }^{2,4}$, Anatolii Bukivskyi ${ }^{1}$, Olexander Vinijchuk ${ }^{5}$, Mykola Shandura ${ }^{5}$, \\ Viktor Yakubovskyi ${ }^{5}$, Yuri Kovtun ${ }^{5}$, Graham A Rance ${ }^{6}$, Michael Fay ${ }^{7}$, Yuri Piryatinski ${ }^{1}$, Oleksiy Kachkovsky ${ }^{5}$, \\ Anatoli Verbitsky ${ }^{1}$ and Aleksey Rozhin ${ }^{2}$
}

The multifunctional properties of carbon nanotubes (CNTs) make them a powerful platform for unprecedented innovations in a variety of practical applications. As a result of the surging growth of nanotechnology, nanotubes present a potential problem as an environmental pollutant, and as such, an efficient method for their rapid detection must be established. Here, we propose a novel type of ionic sensor complex for detecting CNTs - an organic dye that responds sensitively and selectively to CNTs with a photoluminescent signal. The complexes are formed through Coulomb attractions between dye molecules with uncompensated charges and CNTs covered with an ionic surfactant in water. We demonstrate that the photoluminescent excitation of the dye can be transferred to the nanotubes, resulting in selective and strong amplification (up to a factor of 6) of the light emission from the excitonic levels of CNTs in the near-infrared spectral range, as experimentally observed via excitation-emission photoluminescence (PL) mapping. The chirality of the nanotubes and the type of ionic surfactant used to disperse the nanotubes both strongly affect the amplification; thus, the complexation provides sensing selectivity towards specific CNTs. Additionally, neither similar uncharged dyes nor CNTs covered with neutral surfactant form such complexes. As model organic molecules, we use a family of polymethine dyes with an easily tailorable molecular structure and, consequently, tunable absorbance and PL characteristics. This provides us with a versatile tool for the controllable photonic and electronic engineering of an efficient probe for CNT detection.

Light: Science \& Applications (2016) 5, e16028; doi:10.1038/Isa.2016.28; published online 12 February 2016

Keywords: carbon nanotubes; ionic surfactant; organic dye; photoluminescence sensor

\section{INTRODUCTION}

In addition to being one of the best and most readily available onedimensional (1D) model systems, carbon nanotubes (CNTs) demonstrate remarkable potential for multifunctional applications in electronics, reinforced composite materials, chemical and biological sensing, scanning probe microscopy, and many other areas ${ }^{1-6}$. Chemically functionalized CNTs could be designed for various advanced applications, including light-harvesting devices, sensors, drug delivery, and energy storage systems ${ }^{4-10}$. Whereas some of the mentioned applications remain theoretical, others, such as composite frames and coatings and rechargeable batteries, are being realized in practice ${ }^{10}$. However, with the strong, continuous growth of nanotechnology and a total mass production of CNTs exceeding thousands of tons per year ${ }^{10}$, CNTs could represent a significant source of industrial pollution in the near future. This concern is heightened by the fragmentary knowledge available regarding their toxicity ${ }^{8,9,11,12}$. CNTs can escape into the environment during production, application, and recycling; thus, the ambient risk posed by CNTs must be carefully controlled. To this end, we must be well equipped for the rapid detection of CNTs to prevent toxic exposure to the human body and in case of technological catastrophes.

Among the known techniques for chemical sensing, one of the most promising is photoluminescence (PL) by virtue of its high sensitivity, efficiency, and simplicity ${ }^{13,14}$. There are several PL-sensing approaches, categorized on the basis of their responses in terms of absolute intensity, intensity ratio, or redistribution of the PL emission spectrum, change in lifetime, and alteration in anisotropic features. Furthermore, some of these approaches can be beneficially combined, thereby improving sensitivity, selectivity, dynamic range, or other sensor parameters.

The most efficient PL sensing can be achieved through enhancement of the emission in the presence of the compound to be detected ${ }^{13,14}$. The quantum yield of the PL emission of CNTs is low ${ }^{1-6}$, and the PL is quenched by both the bundling of the nanotubes ${ }^{15,16}$ and interactions

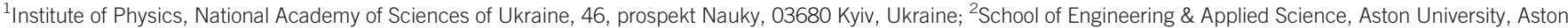

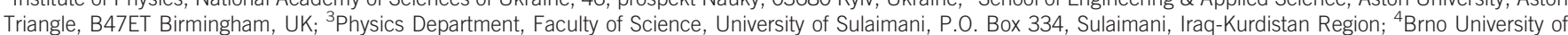

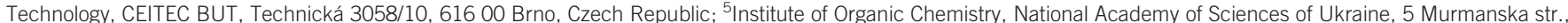

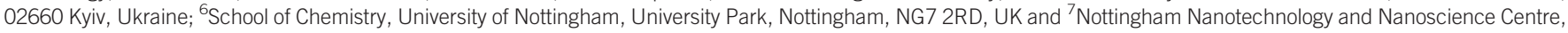
University of Nottingham, University Park, Nottingham, NG7 2RD, UK

Correspondence: A Rozhin, Email: a.rozhin@aston.ac.uk

Received 28 July 2015; revised 11 September 2015; accepted 22 September 2015; accepted article preview online 26 September 2015 
with $\pi$-conjugated compounds and biomaterials ${ }^{17-20}$, for example, rhodamine, fullerenes, or DNA. The "brightening" of CNT PL has been pursued for various photonics applications through the encapsulation of organic materials inside the nanotubes ${ }^{21,22}$, the covalent incorporation of $\mathrm{sp}^{3}$ defects $^{23}$, the embedding of luminescent local states ${ }^{24}$, admixture with an electrolyte ${ }^{25}$, and the non-covalent attachment of $\pi$-conjugated organic molecules ${ }^{26-29}$. The merits of non-covalent attachment of organic molecules include preserving the unique intrinsic features of the nanotubes while simultaneously endowing them with novel advantages associated with the organic conjugates ${ }^{4,8}$. Among the various organic conjugated compounds available, polymethine dyes (PDs), which are organic molecules that contain polymethine chains of variable lengths and terminal functional groups, can be easily modified and tuned for particular opto-electronic tasks ${ }^{30}$. Specifically, the most important distinguishing feature of PDs and their donor-acceptor conjugates is their intense and relatively narrow absorption and fluorescence bands, which can be deliberately tuned in the visible and NIR range through modification of the polymethine chain length or through the attachment of specific terminal groups with their own large $\pi$-electron systems. Furthermore, PDs behave as advanced photonic materials known as spectral sensitizers ${ }^{31}$, light harvesters $^{32}$, and "laser" dyes with large optical nonlinearity ${ }^{33-35}$.

One of our objectives in this study was to pioneer an efficient method for the detection of CNTs through considerable amplification of CNT PL via their interaction with tailorable PDs. In water, CNTs become encompassed by ionic surfactant to form micelles ${ }^{36}$, thus interfering with the interactions between most studied PL probes and the CNTs themselves. Thus, we need to identify an efficient mechanism for sensing CNTs in aqueous media and appropriate probe materials that will help us to overcome the presence of surfactant around the CNTs and thus significantly enhance the sensitivity of the PL sensor.

\section{MATERIALS AND METHODS}

\section{Sample preparation}

Purified single-wall CNTs (CoMoCAT) were purchased from SWeNT, Inc. (SWeNT CG100, Lot \# 000-0012) and used as a source material to prepare the CNT dispersions. In a typical experimental procedure, $1.2 \mathrm{mg}$ of CNTs was dispersed in $20 \mathrm{~mL}$ of deionized water in the presence of a surfactant. The ionic surfactants sodium dodecylbenzene sulfonate (SDBS), sodium dodecylsulfate (SDS), sodium deoxycholate (SDOC), and sodium taurodeoxycholate (STDOC) and the neutral surfactants polyoxyethylene octyl phenyl ether (Triton X-100) and polyvinylpyrrolidone (PVP) were used to disperse the CNTs. We used the following amounts of surfactant in the above dispersions: $6.5 \mathrm{mg}$ of SDBS, SDS, and SDOC; $5.0 \mathrm{mg}$ of STDOC; $15.2 \mathrm{mg}$ of Triton X-100; and $19.2 \mathrm{mg}$ of PVP. The use of higher amounts of the last two surfactants, Triton X-100 and PVP, was motivated by the need to increase the low concentrations of debundled CNTs in the supernatant dispersions. First, the above dispersions were subjected to ultrasonication using a NanoRuptor (Diagenode) processor for $1 \mathrm{~h}$ at $21 \mathrm{kHz}$ and $250 \mathrm{~W}$. Second, the dispersions were subjected to ultracentrifugation for $2 \mathrm{~h} 30 \mathrm{~min}$ at $17^{\circ} \mathrm{C}$ at 47000 rpm for SDBS and $20000 \mathrm{rpm}$ for the other surfactants (Beckman Coulter Optima Max-XP, MLS 50 rotor) to remove the aggregate phase and obtain debundled supernatant solutions of CNTs.

A series of PDs was supplied by the Institute of Organic Chemistry (NASU, Ukraine). The following dye concentrations were studied: $0.0005,0.001,0.002,0.004$, and $0.008 \mathrm{mg} \mathrm{mL}^{-1}$. Mixtures with higher concentrations of PDs $\left(>0.008 \mathrm{mg} \mathrm{mL}^{-1}\right)$ were not considered in the discussion because of the effect of PL emission re-absorbance. To prepare mixtures of CNTs and PDs, we used approximately $20 \%$ of the initial solutions of CNTs dispersed in SDBS and SDOC, approximately $33 \%$ of the initial solutions of CNTs dispersed in STDOC and Triton X-100, and approximately $80 \%$ of the initial solutions of CNTs dispersed in PVP and SDS. The different percentages of the initial CNT solutions were chosen to ensure that the PL signal was compatible with the sensitivity limits of the PL detector. The concentrations of specific CNTs in all mixtures and neat CNTs for the reference media used in the experiments were the same $(20 \%, 33 \%$, and $80 \%$, respectively, for the abovementioned surfactants).

To study the dependence of the PL on the CNT concentration dispersed in SDBS, we tested a range of diluted concentrations (from $3 \%$ to $20 \%$ of the initial CNT concentration).

\section{Experimental setup}

The absorption spectra in the visible and NIR ranges were measured using a Lambda 1050 UV/VIS/NIR (Perkin Elmer) spectrometer. The fluorescence emission spectra at various excitation wavelengths were recorded using a Horiba NanoLog excitation-emission spectrofluorometer equipped with a nitrogen-cooled InGaAs array detector to generate PL excitation-emission maps (PLE maps), with the $X$-axis representing the wavelength of the PL emission, $\lambda_{\mathrm{EM}}$, and the $Y$-axis representing the excitation wavelength, $\lambda_{\mathrm{EX}}$. Entrance/exit slits of $14 \mathrm{~nm}$ in width were used for both the excitation and emission monochromators in the NIR PL measurements. The PL experiments in the visible range were performed using a silicon detector and $2 \mathrm{~nm}$ entrance/exit slits for the monochromators. The time-resolved PL decays were measured using a LifeSpec II spectrofluorometer (Edinburgh Instruments Ltd., UK) to perform time-correlated single-photon counting (TCSPC) under excitation by a picosecond pulsed diode laser at $405 \mathrm{~nm}$.

Raman spectra were recorded using a Horiba-Jobin-Yvon LabRAM Raman microscope with a laser wavelength of $660 \mathrm{~nm}$, operating at a power of approximately $14.5 \mathrm{~mW}$ and with a grating of 600 lines $\mathrm{mm}^{-1}$. A slit of $50 \mu \mathrm{m}$ in width was used to improve the spatial resolution. The detector was a Synapse CCD detector. The spectra were collected by averaging 4 acquisitions, each of $30 \mathrm{~s}$ in duration. The Raman shift was calibrated using the Raleigh peak and the $520.7 \mathrm{~cm}^{-1}$ silicon line from an $\mathrm{Si}(100)$ reference sample. Samples were dropcast from solution onto $\mathrm{Si}(100)$ wafers and dried at room temperature prior to analysis. A minimum of three spectra were recorded from different areas of each sample to ensure statistical relevance.

\section{RESULTS AND DISCUSSION}

By testing a series of newly synthesized and well-known PDs for the rapid recognition of CNTs covered with ionic and non-ionic surfactants in water, we demonstrated enhancement of the PL from the studied semiconductor tubes functionalized by astraphloxin dye (Figure 1). The astraphloxin molecule consists of cyanine terminal groups connected to polymethine chains, and this molecule has an uncompensated positive charge on one of its nitrogens (Figure 2a). We selected SDBS as a basic surfactant in our experiments because of its high efficiency in dispersing CNTs and thus yielding stable debundled nanotubes in water ${ }^{36}$.

In the mixtures of CNTs with dozens of the tested PDs (some of them are described in the Supplementary file), we observed slight quenching of the PL with no influence on the NIR emission of the CNTs. However, astraphloxin resulted in significant amplification of the PL emission from the CNTs, which we attribute to the formation of complexes consisting of CNTs, surfactant, and dye. The behaviour 
a

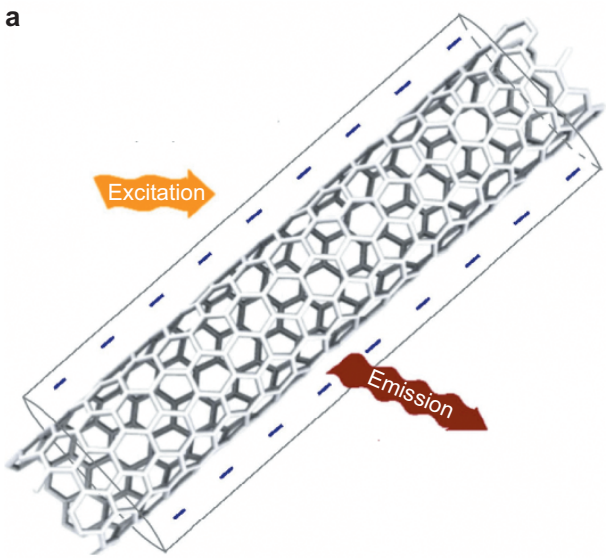

CNT - surfactant b

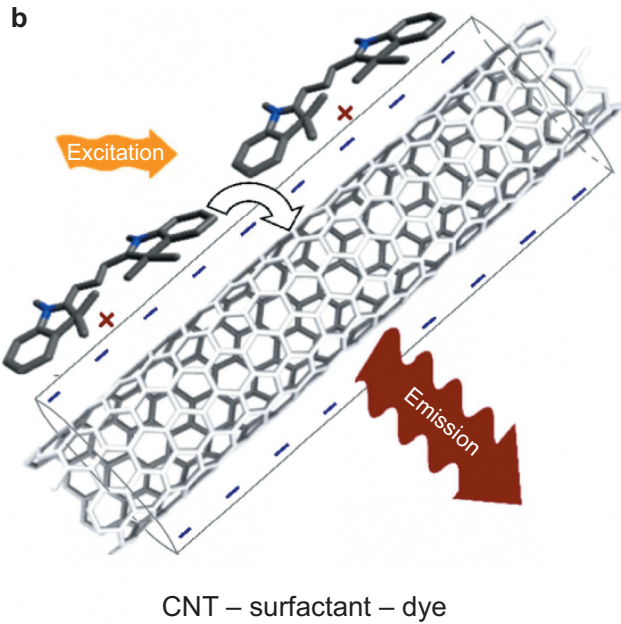

Figure 1 Schematic diagram of energy transfer in the studied CNT-surfactant-dye complexes. (a) A micelle is formed around a CNT by an anionic surfactant. (b) A positively charged astraphloxin molecule becomes attached to the negatively charged CNT micelle through Coulomb attraction, resulting in non-covalent conjugation and enhanced PL emission from the CNT upon excitation of the dye.

observed in the absorption spectra of the studied complexes helped us to understand the nature of this complexation. The maximum of the absorption spectrum for a PD molecule $\left(\lambda_{\mathrm{M}}\right)$ is predominantly determined by the length of the $\pi$-conjugated polymethine chain ${ }^{30}$. In our study, the absorption spectrum of an astraphloxin solution exhibited a maximum at $\lambda_{\mathrm{M}}=540 \mathrm{~nm}$ (Figure 3a, curve 1) regardless of its concentration (Supplementary Figure S1). Because of the complexation of astraphloxin with CNTs, the absorption spectra for the mixtures of astraphloxin with CNTs contained red-shifted dye absorption peaks. The extent of the red-shift depended on the dye concentration (Figure 3a, Supplementary Figure S2). Essentially, the absorption peak of the astraphloxin monomers $\left(\lambda_{\mathrm{M}}\right)$ vanished in the CNT mixtures at low dye concentrations (Figure 3a, curve 2). The red-shifted absorption bands in the range of $560-600 \mathrm{~nm}$ were referenced to J-like aggregates of astraphloxin of various lengths ${ }^{32,37,38}$. J-aggregation is a supramolecular self-organization of particular dyes in solution to form "staircase"-type units ("head-to-tail" aggregation). Note that a
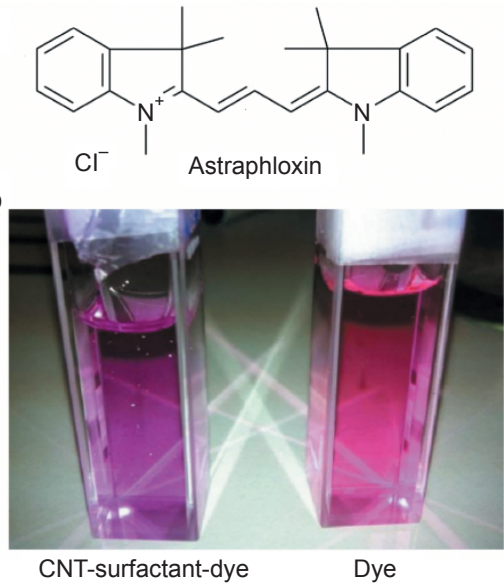

Figure 2 (a) Molecular structure of astraphloxin and (b) photographs of sampling cells with aqueous solutions of neat astraphloxin (right) and a mixture of astraphloxin with CNTs dispersed with SDBS (left), in which small, shiny particles/aggregates (white points) are visible to the naked eye. neat solutions of the dye remained stable for weeks with no aggregate formation, whereas mixtures of astraphloxin with CNTs that were aged overnight (or longer) contained small, shiny crystals/particles (Figure 2b) as well as dye deposited on the walls of the sampling cells. An increase in the dye concentration resulted in an increase in the intensity $\left(\lambda_{\mathrm{M}}\right)$ of the peak associated with the dye monomers (Figure 3a, curves 3 and 4). Mixtures with high concentrations of astraphloxin yielded absorption spectra that could be fitted with a superposition of three components: one associated with the dye monomers, one associated with the interactions between the dye and the CNTs, and one associated with the CNTs themselves (Figure 3a, Supplementary Figure S2). Additionally, the PL excitation spectra (specifically, the dependence of the PL emission at a specific wavelength $\left(\lambda_{\mathrm{EM}}\right)$ on the excitation wavelength $\left.\left(\lambda_{\mathrm{EX}}\right)\right)$ displayed a consistent red-shift of the peak (from 549 to $557 \mathrm{~nm}$, curves 2-4 in Figure $3 \mathrm{~b}$ ) with increasing dye concentration in the mixtures. Thus, the explicit red-shift of the absorption and PL excitation peaks can be explained by the interaction of the PD molecules with the CNT micelles and the formation of J-like aggregates of astraphloxin in the vicinity of the nanotubes. As shown in Figure 3a, in the studied mixtures, we observed a threshold in the appearance of the dye monomers (with $\lambda_{\mathrm{M}}$ ) at concentrations in the range of $0.002-0.004 \mathrm{mg} \mathrm{mL}^{-1}$, indicating saturation in the attachment of the dye molecules to the nanotubes. From this observation, we infer that astraphloxin may cover the surface of a CNT only in a single layer. A similar phenomenon of single-layer formation has been demonstrated in a system of CNTs non-covalently covered with porphyrin molecules ${ }^{28}$.

The interaction of astraphloxin with CNTs is evidenced by the absorbance data in the range of transparency of the dyes (Supplementary Figure S3), where we analysed the excitonic peaks $\mathrm{E}_{11}(900-1250 \mathrm{~nm})$ and $\mathrm{E}_{22}(550-750 \mathrm{~nm})$ in the CNT absorption spectra for the above mixtures. The high-energy $\mathrm{E}_{22}$ peak of the $\mathrm{CNTs}$, with its maximum at $655 \mathrm{~nm}$, does not change in position, whereas the low-energy $\mathrm{E}_{11}$ peaks at 994 and $1024 \mathrm{~nm}$ are red-shifted to 999 and $1029 \mathrm{~nm}$, respectively.

In the PL studies of the mixtures of PDs with CNTs in the range of intrinsic dye emission, we observed significant quenching (by a factor of 4) and red-shifting of the maxima (at approximately $11 \mathrm{~nm}$ ) of the emission of the astraphloxin as a result of its admixture with CNTs 

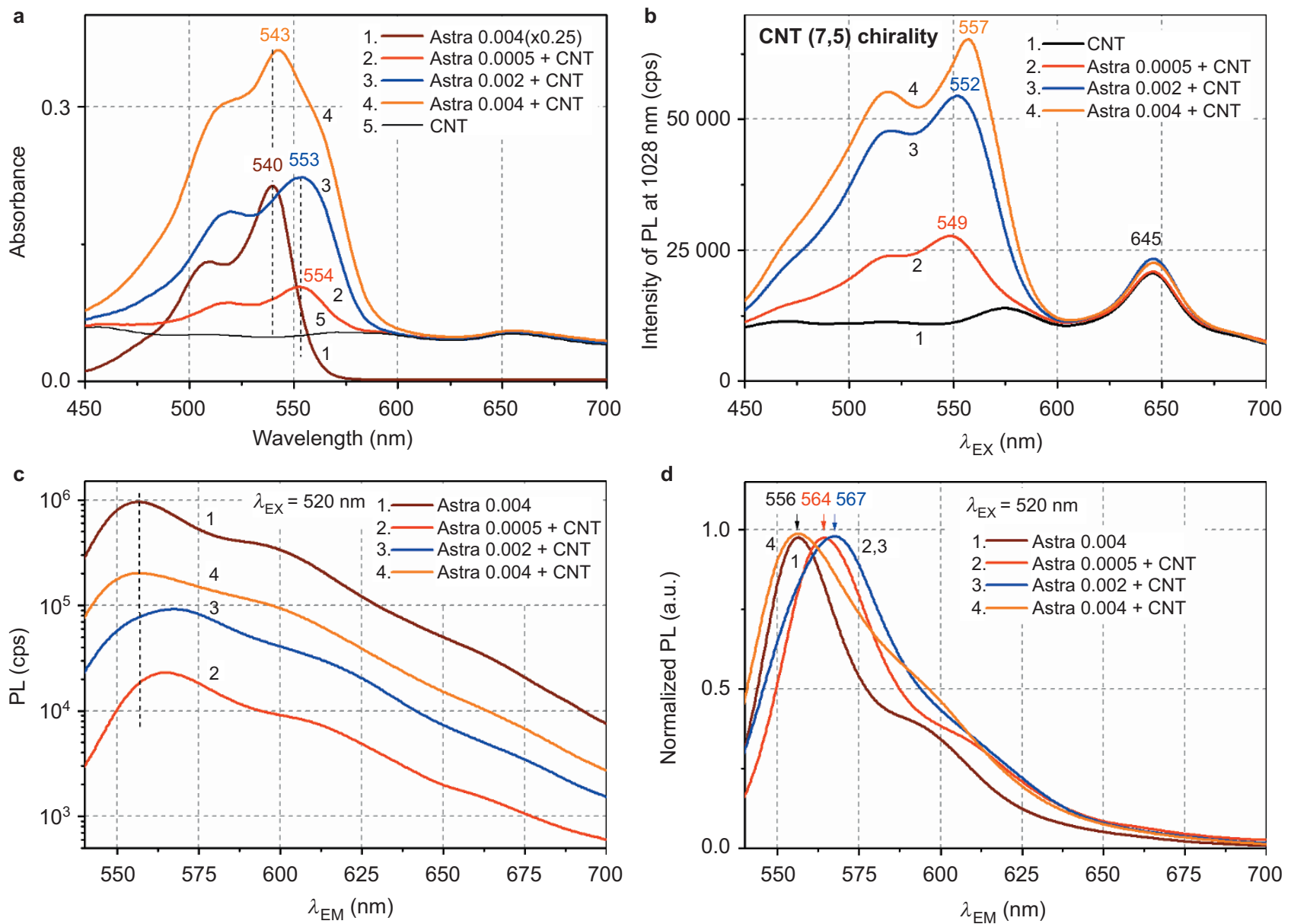

Figure 3 Absorption and PL spectra of astraphloxin and CNTs dispersed with SDBS. (a) Absorption spectra of water solutions of astraphloxin (1-0.004 mg mL ${ }^{-1}$ ), mixtures of CNTs with astraphloxin (2-0.0005 mg mL $-1,3-0.002 \mathrm{mg} \mathrm{mL}^{-1}$, and 4-0.004 mg mL ${ }^{-1}$, where the concentrations given are the dye concentrations in the mixtures), and neat CNTs (5) as a reference; note that curve labels 2-4 correspond to the same dye concentrations throughout Figure 3. (b) PL excitation spectra at $\lambda_{\mathrm{EM}}=1028 \mathrm{~nm}$ for water solutions of neat CNTs (1) and mixtures of CNTs with astraphloxin (2-4); $\lambda_{\mathrm{EM}}=1028 \mathrm{~nm}$ corresponds to $(7,5)$ tube emission. (c, d) PL spectra, (c) as measured and (d) normalized to the maximum, for water solutions of astraphloxin $\left(1-0.004 \mathrm{mg} \mathrm{mL}^{-1}\right.$ ) and mixtures of astraphloxin with CNTs (2-4) at $\lambda_{\mathrm{EX}}=520 \mathrm{~nm}$. Here, "astra" is an abbreviation for astraphloxin and is used in the curve markers for clarity.

(Figure $3 \mathrm{c}$ and $\mathrm{d}$ ). The observed PL quenching can be partially explained by the non-radiative transfer of the excitation energy from the dye to the CNTs, followed by further emission from the CNT excitonic levels. The magnitude of the PL quenching is much lower than that for CNTs covered with porphyrin in the absence of surfactant molecules (this quenching is by three orders of magnitude) ${ }^{28}$. This finding indicates that in the studied complexes, the surfactant remains between the CNT and the dye, limiting the approach distance between them. By fitting the time-resolved PL kinetics in the dye emission range, we obtained a PL lifetime of $30-40$ ps for neat astraphloxin, whereas in the solutions with CNT complexes, this lifetime was supplemented by a second component amounting to $1.5 \mathrm{~ns}$ regardless of the astraphloxin concentration. A lifetime in the range of $1 \mathrm{~ns}$ has been observed for astraphloxin deposited on a substrate as a thin layer ${ }^{37}$. Therefore, the second lifetime component appears to be attributable to the fixation of the dye on the surfaces of the CNT-surfactant micelles. This fixation hinders the rapid oscillations of the astraphloxin molecule and thus delays the relaxation process of PL excitation-emission from the picosecond timescale to the nanosecond timescale.

In the NIR range, we measured PL excitation-emission (PLE) maps for neat CNTs and mixtures of CNTs with various concentrations of astraphloxin (Figure 4, and see Supplementary Figure S4 for more PLE maps). The PLE maps for the neat CNTs (Figure 4a) with $\lambda_{\mathrm{EX}}$ at $\mathrm{E}_{22}, \mathrm{E}_{33}$, and $\mathrm{E}_{44}$ and $\lambda_{\mathrm{EM}}$ at $\mathrm{E}_{11}$ clearly reveal the presence of CNTs with chiralities of $(6,5),(7,5)$, and $(8,4)$ in the studied dispersions. The PLE maps of the mixtures indicate an increase in the PL intensity from tubes of all chiralities $\left(\mathrm{E}^{*}{ }_{11}\right)$ in the dye excitation range, with a maximum at $\lambda_{\mathrm{EX}}=$ $555 \mathrm{~nm}$ (Figure $4 \mathrm{~b}$ ). The maximum of $\lambda_{\mathrm{EX}}$ does not coincide with $\lambda_{\mathrm{M}}$, the absorption maximum of the monomers. However, it does coincide with the absorption maximum for the interaction of the dye with the CNTs (554 nm at $0.0005 \mathrm{mg} \mathrm{mL}^{-1}$ in Figure 3a) and corresponds to $\mathrm{E}_{\text {Dye }}$ in Figure $4 \mathrm{~b}$. The resonant energy transfer (RET) from the astraphloxin-surfactant (donor) complex attached to the CNT (acceptor) is the most obvious cause of this effect and can be explained in terms of dipole-dipole interactions between the molecules. Moreover, the dye monomers present in the mixtures at high concentration do not transfer energy to the CNTs, as seen from the absence of PL peaks at $\lambda_{\mathrm{EX}}=$ $\lambda_{\mathrm{M}}=540 \mathrm{~nm}$ (Figure $4 \mathrm{~b}$ ). This means that short-range energy transfer of the Dexter type is more likely than a Förster mechanism for RET, in which energy can be transferred over distances of up to $90 \AA^{39}$.

To more explicitly demonstrate the amplification of CNT emission, we extracted the PL emission spectra at $\lambda_{\mathrm{EX}}=555 \mathrm{~nm}$ from the PLE 

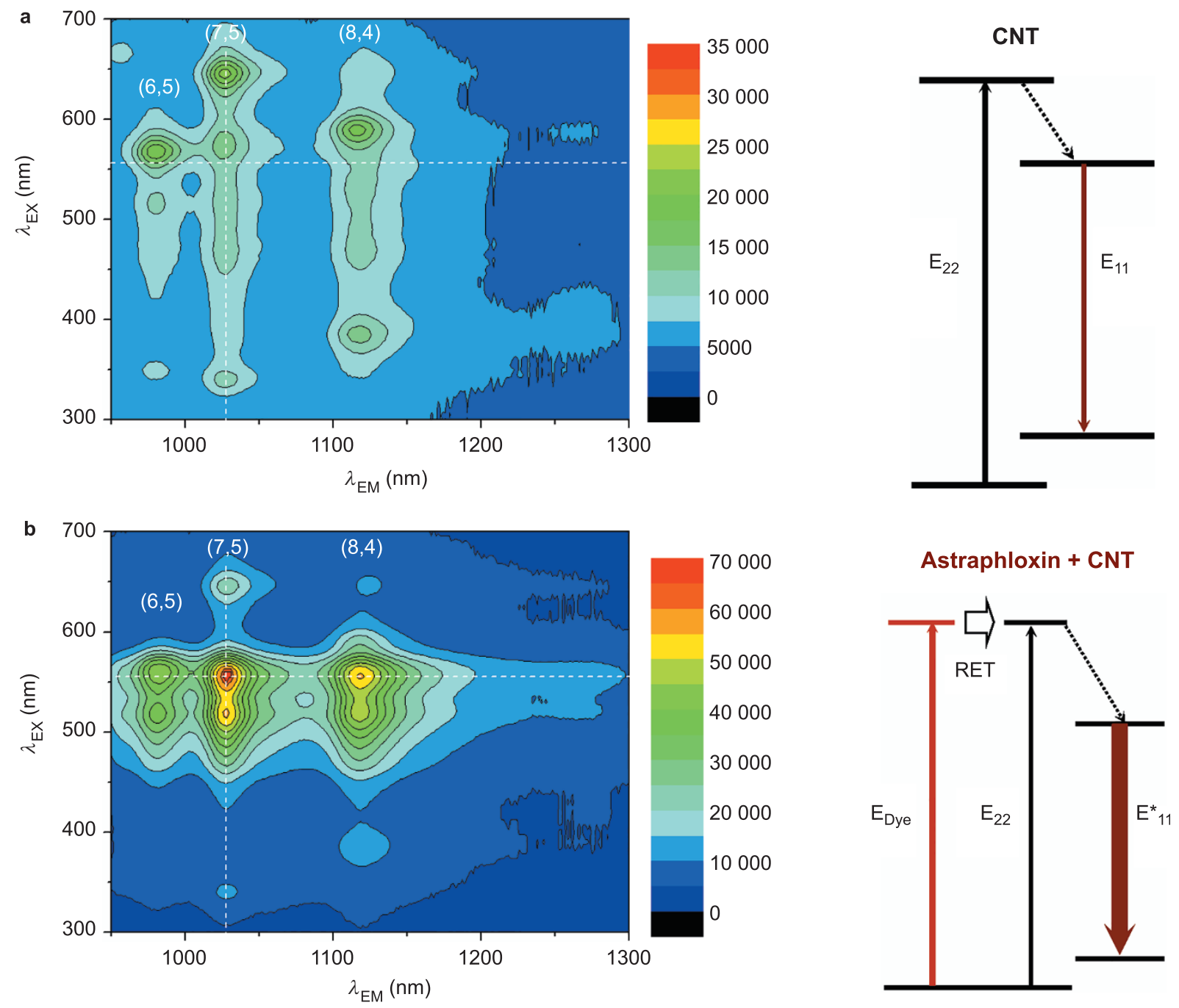

Figure 4 PLE maps for water solutions of (a) neat CNTs dispersed with SDBS and (b) a mixture of CNTs with astraphloxin $\left(0.004 \mathrm{mg} \mathrm{mL}^{-1}\right)$. The left-hand inserts of energy diagrams indicate that (a) CNTs have characteristic exciton energy levels of PL excitation $\left(E_{22}\right)$ and emission ( $\left.E_{11}\right)$ in the visible and NIR ranges, respectively, and $(\mathbf{b})$ a visible-range excitation of the dye molecules ( $E_{\text {Dye }}$ ) attached to the nanotubes is transferred via RET to these CNT levels ( $E_{22}$ with subsequent relaxation to $E_{11}^{*}$ in the NIR range). High PL intensities are coloured red, whereas low intensities are coloured blue (in the electronic version of the manuscript). It should be noted that the PL intensities in part (a) are twofold lower than those in part (b). See Supplementary Figure S5 for more PLE maps.

maps for presentation in Figure 5a. The maximum PL amplification was achieved at an astraphloxin concentration of $0.004 \mathrm{mg} \mathrm{mL}^{-1}$. Moreover, the PL spectra indicate the different effects of the dye on CNTs of each chirality, revealing selectivity in the sensing of CNTs of various diameters (Figure 5b). In Figure 5b, we selected the data from Figure 5 a corresponding to a dye concentration of $0.004 \mathrm{mg} \mathrm{mL}^{-1}$ and to neat CNTs and plotted a histogram of the PL amplification ranges for CNTs of various chiralities - $(6,5),(7,5)$, and $(8,4)$. The highest (up to a factor of 6) PL amplification was achieved for the $(7,5)$ chirality. The varying selectivity for CNT chirality can be explained in terms of factors related to electronic structure and geometry. The latter are related to the diameter of the nanotubes or, more precisely, the surface area of the CNTs to which the dye molecules can become attached. Considering this approach only, a larger surface area implies that a greater number of dye molecules can complex with the CNTs. However, the CNTs of the $(8,4)$ chirality, those with the largest diameter, did not exhibit the highest PL amplification. This finding indicates that the electronic structure parameters of the CNTs and the organic dye must simultaneously influence the PL amplification. Therefore, the structural matching of the $\pi$-electron systems of specific chiral CNTs and organic molecules is of considerable importance in determining the selectivity of non-covalent interactions ${ }^{40}$.

The different dependences of the PL in the visible range (emission from the dye) and the NIR range (emission from the CNTs) on the astraphloxin concentration in the mixture are depicted in Figure 5c. The NIR emission tends to saturate, whereas the PL in the visible range grows until the phenomenon of re-absorbance of the emission begins to hinder this effect. The saturation of the NIR PL suggests that the number of astraphloxin molecules that are able to attach to a CNT is limited. An increase in the dye concentration results in the presence of free dye molecules that do not interact with the CNTs and emit light at the maximum of the monomers (Figure $3 \mathrm{~d}$ ).

We note that the intensity of the intrinsic PL of the CNTs (peak at $\lambda_{\mathrm{EX}}$ $=645 \mathrm{~nm}$ and $\lambda_{\mathrm{EM}}=1028 \mathrm{~nm}$ in Figures $3 \mathrm{~b}$ and 4 ) changes very little and can be regarded as constant with the variation in the dye concentration. Thus, the interactions of the CNTs with the astraphloxin 
a

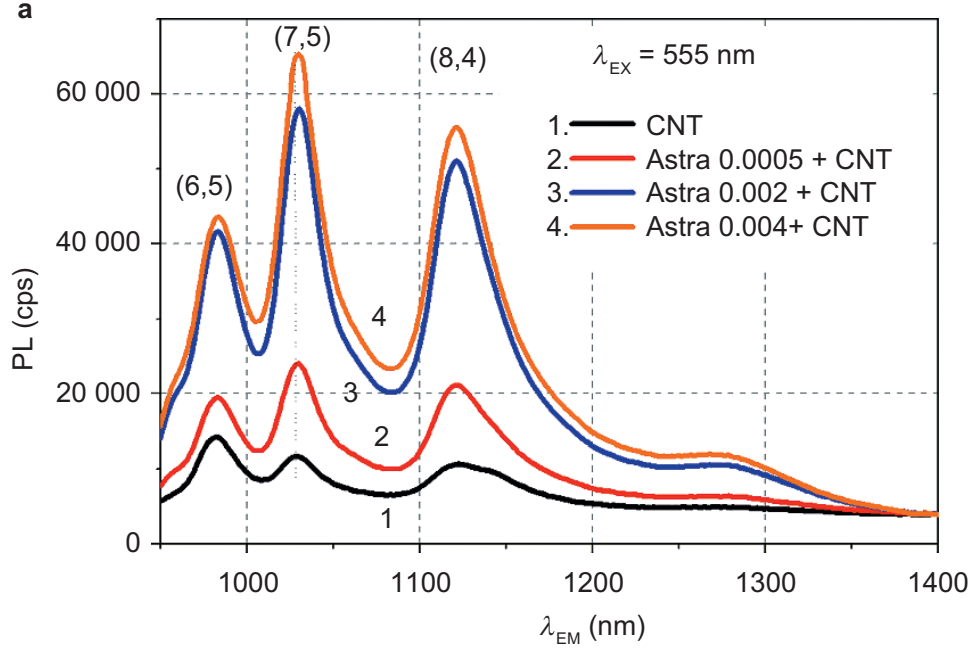

b

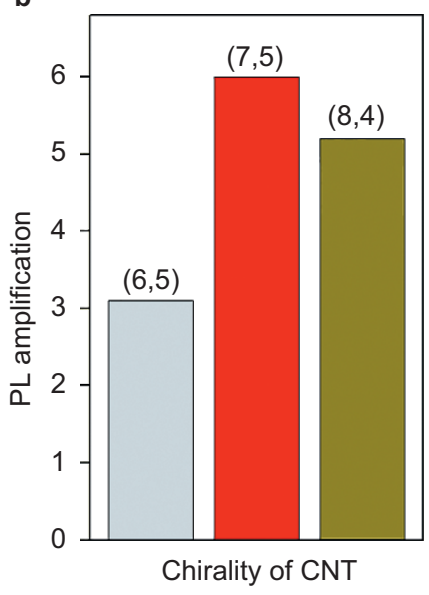

c

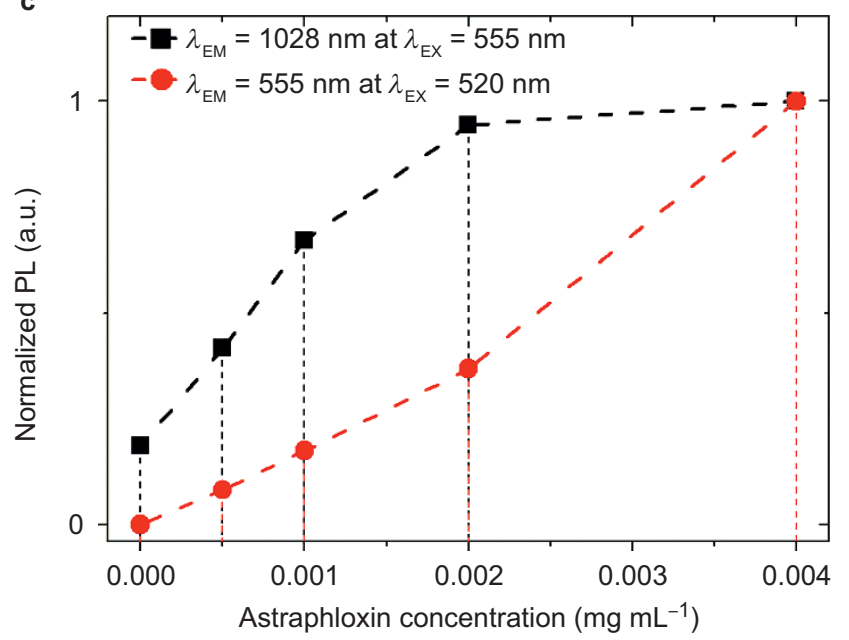

d

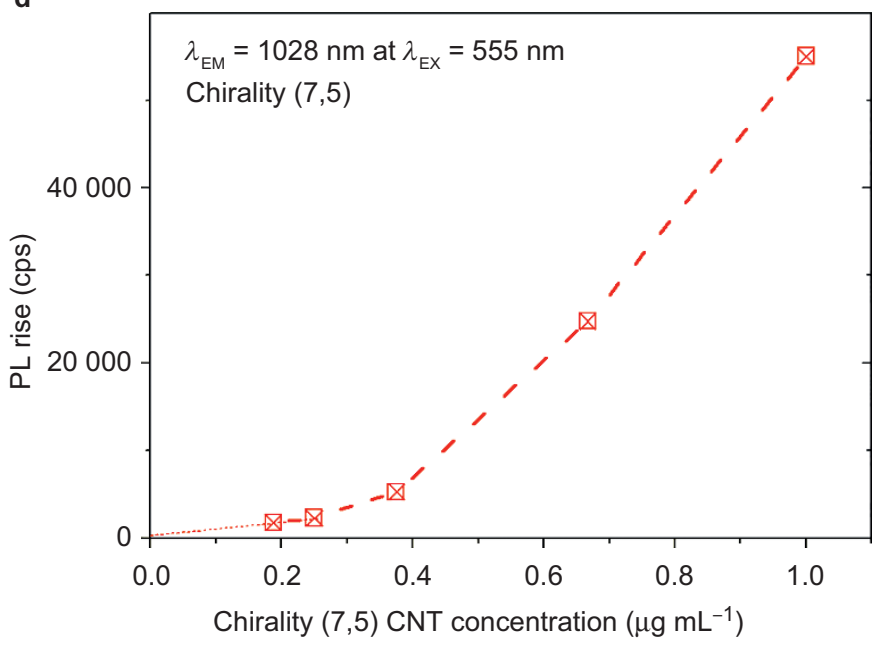

Figure 5 Dependence of PL on astraphloxin concentration in mixtures with CNTs dispersed with SDBS, including the variations in the PL amplification vs. the CNT chirality and the concentrations of the mixtures. (a) PL spectra of water solutions of neat CNTs (1) and mixtures of CNTs with astraphloxin (2-0.0005 mg mL ${ }^{-1}$, 3-0.002 $\left.\mathrm{mg} \mathrm{mL}^{-1}, 4-0.004 \mathrm{mg} \mathrm{mL}^{-1}\right)$. (b) Histogram of the PL amplification in the NIR range $\left(\lambda_{\mathrm{EX}}=555 \mathrm{~nm}\right)$ for astraphloxin $\left(0.004 \mathrm{mg} \mathrm{mL}^{-1}\right)$ with CNTs of various chiralities - (6,5), (7,5), and (8,4). The amplification data are presented as the ratio of curve 4 to curve 1 in Figure 5a. (c) Concentration dependence of the PL for mixtures of CNTs with astraphloxin at $\lambda_{\mathrm{EX}}=555 \mathrm{~nm}$ and $\lambda_{\mathrm{EM}}=1028 \mathrm{~nm}$ (squares) and at $\lambda_{\mathrm{EX}}=520 \mathrm{~nm}$ and $\lambda_{\mathrm{EM}}=555 \mathrm{~nm}$ (circles). (d) The increase in PL in an astraphloxin admixture as a function of the concentration of CNTs with the $(7,5)$ chirality in the SDBS supernatant solution at $\lambda_{\mathrm{EX}}=555 \mathrm{~nm}$ and $\lambda_{\mathrm{EM}}=1028 \mathrm{~nm}$.

sustain their intrinsic PL properties while endowing them with novel advantages. Furthermore, the preservation of the intrinsic features of the CNTs observed in our study is a common phenomenon in the noncovalent functionalization of CNTs by organic molecules ${ }^{4,5,8}$.

To explore the possible application of the studied complexes in sensorics, we analysed the dependence of the PL amplification on the concentrations of nanotubes of the three basic chiralities (Figure $5 \mathrm{~d}$, and see Supplementary Figure S5 for a description of the estimation of the CNT concentrations). Regarding this concentration dependence, in addition to establishing the shape of the calibration curves for CNT sensing, we evaluated the detection limit, which was found to lie in the range of (1-4) ng mL ${ }^{-1}$ for chiralities of $(6,5),(7,5)$ and $(8,4)$ (see the Supplementary file for details). This detection limit is comparable to that previously obtained by another research group ${ }^{41}$.

Modification of the molecular structure of astraphloxin can help us to understand the specific nature of the CNT-PD interaction and, potentially, to improve the PL amplification. A version of the astraphloxin molecule modified with a long alkyl chain, Cy3, exhibited behaviour similar to that of astraphloxin, but the PL amplification effect was lower, yielding a maximum amplification of a factor of 4 at a Cy3 concentration of $0.002 \mathrm{mg} \mathrm{mL}^{-1}$ (Supplementary Figures S4, S6, and S7). This means that more astraphloxin molecules could contribute to the RET because of the possibility of better regularity in their attachment to the CNTs.

Another investigated approach was to test the PL sensing properties of astraphloxin molecules substituted with different anionic groups. The specific purpose of the test was to exploit PDs with the same molecular backbone modified by an anionic-linking group and thus to neutralize the positive charge of the astraphloxin (i.e., the formation of betaine systems ${ }^{42}$ ). In the absorption and PL spectra, the mixtures of the betaine dyes, namely neutral forms of modified astraphloxin or Cy3, with CNTs resulted in the simple superposition of the two compounds without any new features or PL amplification (see the molecular structures of some of these dyes in Supplementary Figure S8). Thus, we demonstrated that astraphloxin analogues neutralized with anionic groups do not transfer energy to CNTs dispersed with SDBS and that 
there is little or no interaction between such a neutral dye and a charged CNT micelle.

The next logical step was to consider at the effects of various surfactants used to disperse the CNTs on the complexation and, perhaps, PL amplification. We tested CNT micelles dispersed with the nonionic surfactants Triton X-100 and PVP and compared their interactions with astraphloxin and its analogues. In the case of the mixtures of the nanotubes with astraphloxin, a superposition of the spectra of the mixed components was observed and the PL signal of the CNTs decreased in the presence of the dye (Supplementary Figure S9, Supplementary Tables S1-S3). Furthermore, no RET to the nanotubes was observed.

It should be emphasized that the astraphloxin analogues neutralized with anionic groups did not transfer energy to the CNTs regardless of the anionic or neutral nature of the surfactants used. These findings help us to understand the nature of the interaction between the molecules and reveal the mechanism of the complexation between astraphloxin and CNTs. In this manner, the Coulomb attraction of positively charged PDs to anionic groups of the surfactant that forms the CNT micelles is established as a key requirement for the formation of energy transfer complexes and the aggregation of the dyes on CNT micelles. Thus, the utilization of the interactions between charged PDs and surfactant-CNT micelles can be regarded as a novel approach to creating efficient PL sensors for CNTs in aqueous environments.

A series of CNT dispersions with various ionic surfactants (SDBS, SDS, SDOC, and STDOC) were tested for complexation with astraphloxin. A comparison of the absorption spectrum of a neat solution of astraphloxin (with constant $\lambda_{\mathrm{M}}$ ) with those of its mixtures with different CNT micelles revealed the emergence of new absorption features of the mixtures in the range of 550-600 nm (Figure 6a). A new explicit peak at $576 \mathrm{~nm}$ appeared in the absorption spectra of the mixtures with CNTs dispersed with SDOC and STDOC, whereas the spectra of the mixtures with CNTs dispersed with SDBS and SDS exhibited a shoulder in that range. The peak at $576 \mathrm{~nm}$ is attributed to the longer shift (up to $36 \mathrm{~nm}$ ) in absorption that occurs when the dye interacts with CNTs covered with deoxycholate surfactants (SDOC and STDOC). As a result, the absorption peaks of the astraphloxin monomers and astraphloxin-CNT complexes do not overlap in the case of deoxycholate-treated CNTs. This longer shift can be explained in terms of a better arrangement of the astraphloxin molecules in the J-like aggregation because of the influence of the $\pi$-electron systems in the SDOC and STDOC surfactants. For excitation in the range of the new absorption peak (550-600 nm), we observed enhancement of the PL from the CNT levels $\left(E^{*}{ }_{11}\right.$ in Figure $\left.4 \mathrm{~b}\right)$ for all mixtures containing anionic surfactants (Supplementary Figure S9, Supplementary Table S3). The excitation maximum for the PL amplification varied from $\lambda_{\mathrm{EX}}=555 \mathrm{~nm}$ for SDBS to $\lambda_{\mathrm{EX}}=565 \mathrm{~nm}$ for SDS and to $\lambda_{\mathrm{EX}}=570 \mathrm{~nm}$ for SDOC and STDOC. CNTs with the $(7,5)$ chirality displayed the maximum PL amplification for all anionic surfactants. In the histogram presented in Figure $6 \mathrm{~b}$, we summarize the data for the PL enhancement of $(7,5) \mathrm{CNTs}$, including the reduction of the PL intensity for CNTs dispersed with neutral surfactants due to the bundling process mentioned above. The maximum PL enhancement was observed for mixtures of astraphloxin with CNTs dispersed with SDBS (see Supplementary Tables S1-S3 for more data). Thus, this study demonstrated the different effects of the dyes on CNTs of each chirality, revealing selectivity in the sensing of CNTs of various diameters. The effect is similar to the selectivity observed when sorting CNTs using SDOC and STDOC ${ }^{43}$. Additionally, we observed a striking selectivity in PL amplification among CNT-dispersing surfactants.

To confirm that the changes observed in the above systems were caused by the interaction of the PDs with the CNTs, we studied a twocomponent system of mixtures consisting only of the dye (astraphloxin or its analogues) and the surfactant. No PL features appeared in the NIR range of the spectra. Thus, the fundamental contribution to the amplification in the NIR range stems from the interaction of the surfactant-covered CNTs with the dye molecules.

In addition, we performed an alternative characterization of the studied systems via Raman spectroscopy to gain a better understanding of the PL amplification mechanism and the role of ionic and nonionic surfactants in the astraphloxin-CNT interaction. The most important feature of the acquired Raman spectra was an enhancement in the dye bands (relative to the vibrational modes of the CNTs themselves) at $1122,1238,1272,1373,1405$, and $1473 \mathrm{~cm}^{-1}$, which was assigned to the cyanine chromophore and polymethine chain modes ${ }^{44}$, in the mixture of CNTs with ionic SDBS compared with the dye-containing mixture of CNTs with non-ionic Triton X-100 (Figure 7, and see Supplementary Figure S10 for an extension of the
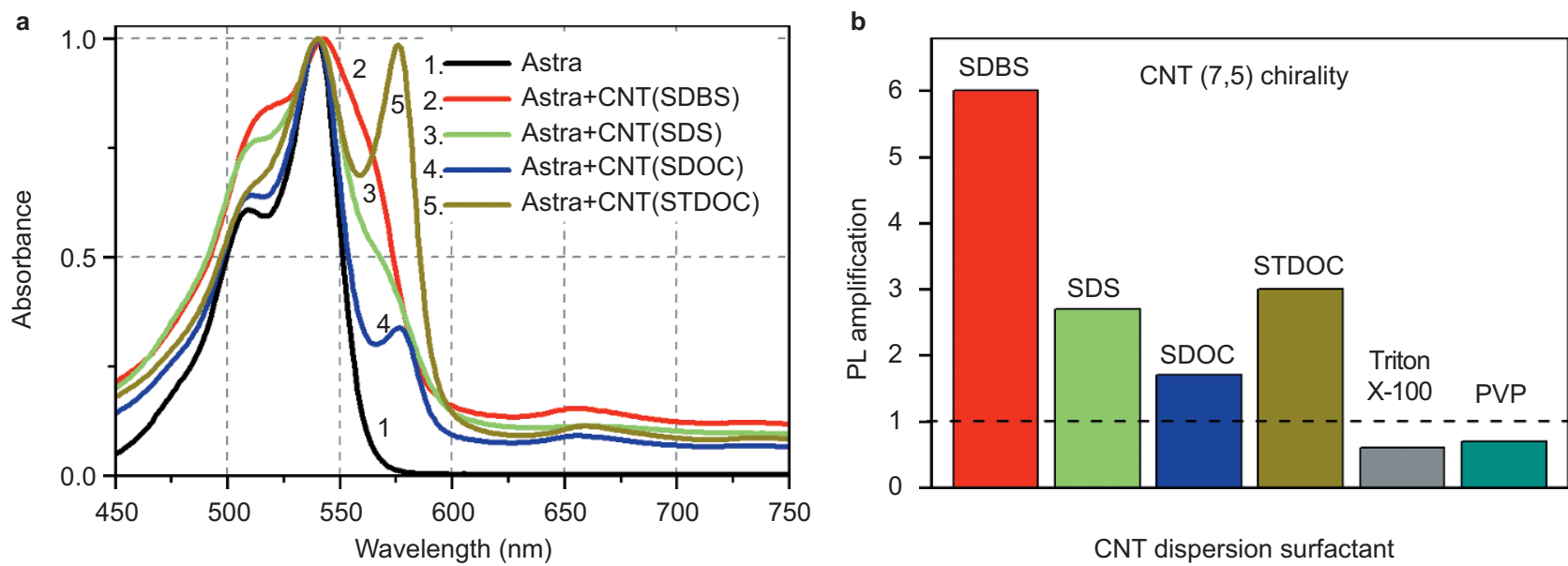

CNT dispersion surfactant

Figure 6 Absorption spectra of astraphloxin with CNTs dispersed with various surfactants. (a) Absorption spectra for water solutions of neat astraphloxin (1) and mixtures of astraphloxin with CNTs dispersed with various surfactants: (2) SDBS, (3) SDS, (4) SDOC, and (5) STDOC. All spectra are normalized to the maximum at approximately $540 \mathrm{~nm}$. (b) Histogram of the PL amplification $\left(\lambda_{\mathrm{EX}}=555-570 \mathrm{~nm}\right)$ in the NIR range for astraphloxin with $(7,5)$ CNTs dispersed with various surfactants - SDBS, SDS, SDOC, STDOC, Triton X-100, and PVP. The astraphloxin concentration in all solutions was $0.004 \mathrm{mg} \mathrm{mL}^{-1}$. 


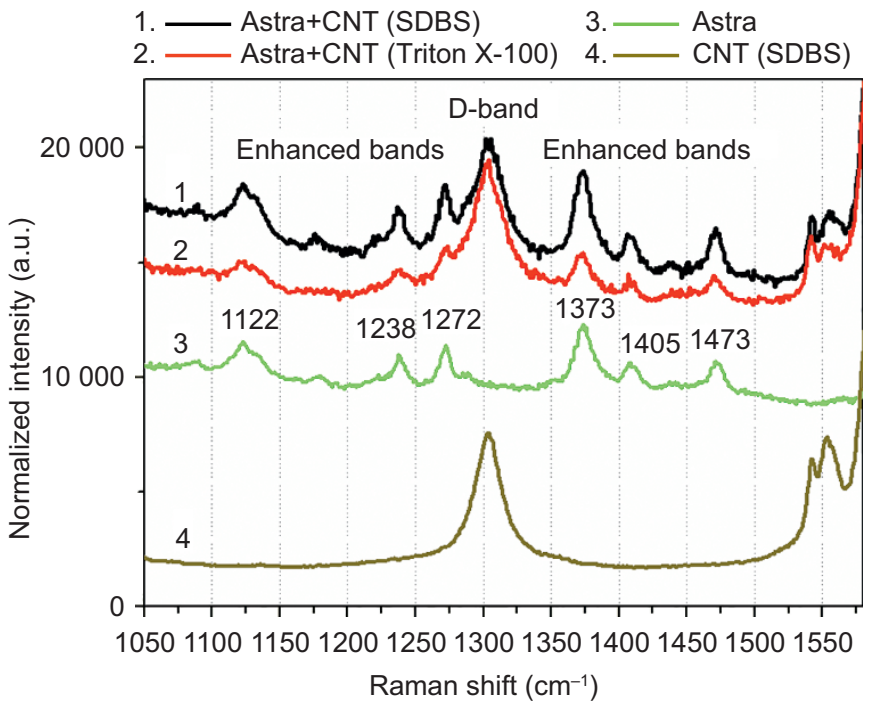

Figure 7 Raman spectra for samples of astraphloxin with CNTs dispersed with SDBS (1) or Triton X-100 (2), neat astraphloxin (3), and CNTs dispersed with SDBS (4). The spectra are normalized to the $G$ band for comparison and offset from each other on the $Y$-axis for clarity. The astraphloxin concentration was $0.004 \mathrm{mg} \mathrm{mL}^{-1}$.

spectral range up to $2750 \mathrm{~cm}^{-1}$ and additional discussion). We attribute the enhancement of the Raman bands to aggregation of the dye molecules, as demonstrated by Akins et al. (for example ${ }^{45}$ ) and as discussed above for ionic complexes.

RET from the dyes aggregated with the CNTs is the most obvious explanation for the PL amplification effect. Thus far, because CNTs and PDs are $\pi$-electron systems, the most feasible form of the interaction between them is an aromatic stacking interaction, as previously discussed in the scientific literature $27,28,46,47$. By means of preliminary quantum-chemical modelling (using the ZINDO/S method), we demonstrated that the interaction between a CNT and a PD molecule separated by a surfactant molecule should be decreased but still present. Strictly speaking, the features of the frontier levels directly affect the lowest electron transitions in CNTs $\left(\mathrm{E}_{11}\right)$ and in CNT-PD complexes. Our model shows that as a result of the complexation of a CNT with astraphloxin, the highest occupied molecular orbital (HOMO) and lowest unoccupied molecular orbital (LUMO) are located on the CNT and the astraphloxin, respectively. Thus, in the CNT-PD complex, a new frontier LUMO emerges that is not present in the pure CNT. In this manner, the features of the frontier orbitals determine the electron transitions corresponding to absorption/fluorescence in the visible and NIR range of the complex system. Thus, the electronic transitions between the LUMO on the astraphloxin and the HOMO on the CNT ( $E^{*}{ }_{11}$ in Figure $4 \mathrm{~b}$ ) in the studied complexes could be responsible for the strong PL amplification observed.

\section{CONCLUSIONS}

We describe a new photonic nanosystem consisting of PDs attached to CNT-surfactant micelles in water, which results in significant amplification of the CNT PL emission. The Coulomb attraction between the dye and surfactant molecules covering the CNTs is key to the emergence of novel photonic complexes. We observe that the creation of such complexes occurs through the Coulomb coupling of the positively charged astraphloxin molecules with the anionic surfactants covering the CNTs. The astraphloxin attached to a micelle interacts with the CNT via weak $\pi-\pi$ complexation, resulting in quenching of the dye emission and enhancement of the CNT PL. PL excitationemission mapping in the spectral ranges of the dye and CNTs allowed us to experimentally demonstrate that only PD molecules associated with nanotube-surfactant micelles contribute to the PL amplification of the CNTs. Moreover, we observed firm evidence of the J-like aggregation of astraphloxin on anionic CNT micelles, supported by absorbance, PL, and Raman spectroscopy measurements. Reference systems with neutral surfactants or modified astraphloxins with negatively charged sulfonated groups were unable to form such complexes with CNTs. We also report the physicochemical model of the complexation process, which is fully supported by a complementary study of the reference systems.

In this paper, we propose an efficient molecular-engineering approach to the detection of CNTs and establish a mechanism for the selective PL sensing of CNTs by means of ionic complexes. It should be emphasized that the limit of CNT detection estimated based on the dependence of the PL amplification on the concentrations of CNTs of various chiralities is in the range of $\mathrm{ng} \mathrm{mL}^{-1}$. The most prominent feature of PDs is their vast variety of functional versatility, including polymethine chain elongation and the possibility of applying various anions/cations to neutralize the charged dyes. We can exploit the easy tailoring of the molecular structures of PDs, which offers excellent prospects for the optimization of the chemical and physical properties of complexes of CNTs and organic dyes with tunable optical properties and thus demonstrates great potential for application in chemical sensing and other photonic applications.

The developed macromolecular CNT-PD complexes provide an innovative means of functionalizing carbon nanomaterials, particularly with the purpose of strong enhancement of PL emission, thereby extending the multifunctional applications of these materials in electronics, ultra-fast photonics, reinforced composite materials, chemical and biological sensing, scanning probe microscopy, drug delivery, energy storage, and many other areas.

\section{ACKNOWLEDGEMENTS}

The work was supported by the NATO SPS Programme (NUKR.SFPP 984189 project). R.A. acknowledges support from the Human Capacity Development Program of the Ministry of Higher Education and Scientific Research of the Iraq-Kurdistan Region. The authors would like to thank the Nottingham Nanotechnology and Nanoscience Centre for access to facilities.

1 Wong HSP, Akinwande D. Carbon Nanotube and Graphene Device Physics. Cambridge: Cambridge University Press; 2011.

2 Hirsch A. The era of carbon allotropes. Nat Mater 2010; 9: 868-871.

3 Hasan T, Sun ZP, Wang FQ, Bonaccorso F, Tan PH et al. Nanotube-polymer composites for ultrafast photonics. Adv Mater 2009; 21: 3874-3899.

4 Meng LJ, Fu CL, Lu QH. Advanced technology for functionalization of carbon nanotubes. Prog Nat Sci 2009; 19: 801-810.

5 Balasubramanian K, Burghard M. Chemically functionalized carbon nanotubes. Small 2005; 1 : 180-192.

6 Avouris P, Freitag M, Perebeinos V. Carbon-nanotube photonics and optoelectronics. Nat Photonics 2008; 2: 341-350.

7 Peng $\mathrm{XH}$, Wong SS. Functional covalent chemistry of carbon nanotube surfaces. Adv Mater 2009; 21: 625-642.

8 Lee Y, Geckeler KE. Carbon nanotubes in the biological interphase: the relevance of noncovalence. Adv Mater 2010; 22: 4076-4083.

9 Vardharajula S, Ali SZ, Tiwari PM, Eroğlu E, Vig K et al. Functionalized carbon nanotubes: biomedical applications. Int J Nanomedicine 2012; 7: 5361-5374.

10 De Volder MFL, Tawfick SH, Baughman RH, Hart AJ. Carbon nanotubes: present and future commercial applications. Science 2013; 339: 535-539.

11 Hussain SM, Braydich-Stolle LK, Schrand AM, Murdock RC, Yu KO et al. Toxicity evaluation for safe use of nanomaterials: recent achievements and technical challenges. Adv Mater 2009; 21: 1549-1559. 
12 Kostarelos K, Bianco A, Prato M. Promises, facts and challenges for carbon nanotubes in imaging and therapeutics. Nat Nanotechnol 2009; 4: 627-633.

13 Demchenko AP. Introduction to Fluorescence Sensing. Berlin: Springer; 2009.

14 Wolfbeis OS. Fluorescence Methods and Applications: Spectroscopy, Imaging, and Probes. Boston: Wiley-Blackwell; 2008.

15 Bergin SD, Nicolosi V, Cathcart H, Lotya M, Rickard D et al. Large populations of individual nanotubes in surfactant-based dispersions without the need for ultracentrifugation. J Phys Chem C 2008; 112: 972-977.

16 Tan PH, Rozhin AG, Hasan T, Hu P, Scardaci V et al. Photoluminescence spectroscopy of carbon nanotube bundles: evidence for exciton energy transfer. Phys Rev Lett2007; 99: 137402.

17 Barone PW, Baik S, Heller DA, Strano MS. Near-infrared optical sensors based on single-walled carbon nanotubes. Nat Mater 2005; 4: 86-92.

$18 \mathrm{Kim} \mathrm{JH}$, Heller DA, Jin H, Barone PW, Song C et al. The rational design of nitric oxide selectivity in single-walled carbon nanotube near-infrared fluorescence sensors for biological detection. Nat Chem 2009; 1: 473-481.

19 Ahmad A, Kurkina T, Kern K, Balasubramanian K. Applications of the static quenching of rhodamine B by carbon nanotubes. ChemPhysChem 2009; 10: 2251-2255.

20 Nismy NA, Jayawardena KDGI, Adikaari AADT, Silva SRP. Photoluminescence quenching in carbon nanotube-polymer/fullerene films: carbon nanotubes as exciton dissociation centres in organic photovoltaics. Adv Mater 2011; 23: 37963800.

21 Yanagi K, lakoubovskii K, Kazaoui S, Minami N, Maniwa Y et al. Light-harvesting function of $\beta$-carotene inside carbon nanotubes. Phys Rev B 2006; 74: 155420.

22 Yanagi $\mathrm{K}$, lakoubovskii $\mathrm{K}$, Matsui $\mathrm{H}$, Matsuzaki $\mathrm{H}$, Okamoto $\mathrm{H}$ et al. Photosensitive function of encapsulated dye in carbon nanotubes. J Am Chem Soc 2007; 129 4992-4997.

23 Piao YM, Meany B, Powell LR, Valley N, Kwon $\mathrm{H}$ et al. Brightening of carbon nanotube photoluminescence through the incorporation of sp3 defects. Nat Chem 2013; 5 840-845.

24 Miyauchi Y, Iwamura M, Mouri S, Kawazoe T, Ohtsu M et al. Brightening of excitons in carbon nanotubes on dimensionality modification. Nat Photonics 2013; 7: 715-719.

25 Duque JG, Oudjedi L, Crochet JJ, Tretiak S, Lounis B et al. Mechanism of electrolyteinduced brightening in single-wall carbon nanotubes. J Am Chem Soc 2013; 135: 3379-3382.

26 Casey JP, Bachilo SM, Weisman RB. Efficient photosensitized energy transfer and near-IR fluorescence from porphyrin-SWNT complexes. J Mater Chem 2008; 18 1510-1516.

27 Ahmad A, Kern K, Balasubramanian K. Selective enhancement of carbon nanotube photoluminescence by resonant energy transfer. ChemPhysChem 2009; 10: 905909.

28 Roquelet C, Langlois B, Vialla F, Garrot D, Lauret JS et al. Light harvesting with non covalent carbon nanotube/porphyrin compounds. Chem Phys 2013; 413: 45-54.

29 Ernst F, Heek T, Setaro A, Haag R, Reich S. Energy transfer in nanotube-perylene complexes. Adv Funct Mater 2012; 22: 3921-3926.

30 Strekowski L. Heterocyclic Polymethine Dyes: Synthesis, Properties and Applications, Series: Topics in Heterocyclic Chemistry, Vol. 14. Berlin: Springer; 2008.

31 Kachkovski AD. Polymethine dyes. Kirk-Othm Encycl Chem Technol 2000; doi: 10.1002/0471238961.1615122511010308.a01.
32 Eisele DM, Cone CW, Bloemsma EA, Vlaming SM, van der Kwaak CGF et al. Utilizing redox-chemistry to elucidate the nature of exciton transitions in supramolecular dye nanotubes. Nat Chem 2012; 4: 655-662.

33 Hermann JP, Ducuing J. Third-order polarizabilities of long-chain molecules. J App Phys 1974; 45: 5100-5102.

34 Hales JM, Matichak J, Barlow S, Ohira S, Yesudas K et al. Design of polymethine dyes with large third-order optical nonlinearities and loss figures of merit. Science 2010; 327: $1485-1488$.

35 Mukhopadhyay S, Risko C, Mardera SR, Brédas JL. Polymethine dyes for all-optical switching applications: a quantum-chemical characterization of counter-ion and aggregation effects on the third-order nonlinear optical response. Chem Sci 2012 3: 3103-3112.

36 Islam MF, Rojas E, Bergey DM, Johnson AT, Yodh AG. High weight fraction surfactant solubilization of single-wall carbon nanotubes in water. Nano Lett2003; 3: 269-273.

37 Piryatinski YP, Furier MS, Nazarenko VG. Electrooptical properties of liquid crystal n-pentil-ń-cyanobifenil with J-aggregates of astrafloxine. Semicond Phys Quant Elect Optoelectr 2001; 4: 375-382.

38 Piryatinskii YP, Nazarenko VG, Yatsun OV. Controllable electrochromic effect in a liquid-crystal cell with J aggregates. Techn Phys Lett 1999; 25: 761-762.

39 Lakowicz JR. Principles of Fluorescence Spectroscopy. New York: Kluwer Academic Plenum Publishers; 1999.

40 Chen Y, Ng AK, Bai S, Si R, Wei L et al. Separation of metallic and semiconducting single-wall carbon nanotubes. In: Zhang Q, editor. Carbon Nanotubes and Their Applications. Stanford: Pan Stanford Publishing Pte. Ltd.; 2012. pp 121-148.

41 Schierz A, Parks AN, Washburn KM, Chandler GT, Ferguson PL. Characterization and quantitative analysis of single-walled carbon nanotubes in the aquatic environment using near-infrared fluorescence spectroscopy. Environ Sci Technol 2012; 46: 12262-12271.

42 Ollis WD, Stanforth SP, Ramsden CA. Heterocyclic mesomeric betaines. Tetrahedron 1985; 41: 2239-2329.

43 Arnold MS, Green AA, Hulvat JF, Stupp SI, Hersam MC. Sorting carbon nanotubes by electronic structure using density differentiation. Nat Nanotechnol 2006; 1: 60-65.

44 Sato H, Kawasaki M, Kasatani K, Katsumata M. Raman spectra of some indo-, thiaand selena-carbocyanine dyes. J Raman Spectrosc 1988; 19: 129-132.

45 Akins DL. Enhanced Raman scattering by molecular nanoaggregates. Nanomater Nanotechnol 2014; 4: 1-17.

46 Zhang Y, Yuan SL, Zhou WW, Xu JJ, Li Y. Spectroscopic evidence and molecular simulation investigation of the $\pi-\pi$ Interaction between pyrene molecules and carbon nanotubes. J Nanosci Nanotechnol 2007; 7: 2366-2375.

47 Pan B, Xing BS. Adsorption mechanisms of organic chemicals on carbon nanotubes. Environ Sci Technol 2008; 42: 9005-9013.

\section{(c) (i) $(-)$ This work is licensed under a Creative Commons Attribution-}

(c) party material in this article are included in the article's Creative Commons license, unless indicated otherwise in the credit line; if the material is not included under the Creative Commons license, users will need to obtain permission from the license holder to reproduce the material. To view a copy of this license, visit http://creativecommons.org/licenses/ by-nc-nd/4.0/ 